\title{
Antiretroviral Drug Substitution Practice in the Treatment Of HIV/TB Co- Infection
}

\author{
${ }^{1}$ Godwin .I. Achinge, ${ }^{1}$ Peter T. Mbaave, ${ }^{2}$ Terkaa .T. Bitto, ${ }^{3}$ Vivian .N. Shaahu, \\ ${ }^{4}$ Hameed Mohammed, ${ }^{5}$ Jonah O. Abah \\ ${ }^{I}$ Department of Medicine, Benue State University Teaching Hospital, Makurdi, Nigeria \\ ${ }^{2}$ Riverside Specialist Clinic, Federal Medical Centre, Makurdi, Benue State, Nigeria \\ ${ }^{3}$ Department of Community Medicine, Federal Medical Centre, Makurdi, Nigeria \\ ${ }^{4}$ Department of Radiology, Benue State University Teaching Hospital, Makurdi, Nigeria \\ ${ }^{5}$ Department of Family Medicine, Federal Medical Centre, Makurdi, Nigeria
}

\begin{abstract}
BACKGROUND: This was a study to evaluate antiretroviral drug substitution practice in the treatment of HIV/TB co-infection.

METHODS: The study was a retrospective review of HIV patients who were treated for tuberculosis over a 2 year period at the HIV clinic of the Federal Medical Centre, Makurdi. All patients who registered for HIV services at that period were reviewed. The case notes of those patients who were treated for tuberculosis during the study period were retrieved, data extracted for analysis using SPSS version 16. Case notes with insufficient information were excluded.

RESULTS: 450 patients were screened for TB, 75 had active TB giving a TB prevalence of 16.7\%. One third (33.3\%) were aged 30-39 years, 22 (29.3\%) were 40-49 years, $17(22.7 \%)$ were <30years and $11(14.7 \%)$ were $\geq 50 y e a r s$ old. Majority 46 (61.3\%) of the patients were females. Over half $41(54.7 \%)$ had secondary or tertiary education and were single, divorced, separated or widowed. Only 28 (37.3\%) were on Efavirenz, an equal number 27 (36\%) were on a Nevirapine while 2 (2.7\%) were on a Protease Inhibitor.

CONCLUSION: This study showed poor compliance with Nigerian treatment protocol for TB/HIV coinfection. Effort to improve TB control in HIV patients are urgently needed
\end{abstract}

\section{KEY WORDS: Antiretroviral, HIV/TB, Co-infection, substitution, practice}

\section{INTRODUCTION}

Tuberculosis (TB) remains a serious public health problem in Nigeria. ${ }^{1}$ The country has the largest TB burden in Africa and ranks 5th among the 22 high TB burden countries which collectively bear $80 \%$ of the global burden of TB. ${ }^{2,3}$ Worldwide, there has been a surge in the occurrence of TB with the advent of HIV in early 1980s. ${ }^{4,5}$ It is a common saying that TB follows HIV as a shadow and TB is the most common and preventable opportunistic infection in HIV-infected patients especially in Africa. The increasing HIV burden in sub-Saharan African countries like Nigeria which saw HIV sero-prevalence rising from 1.8\% in 1991 to $4.1 \%$ in 2010 would imply that the occurrence of TB among HIV patients would continue to increase. ${ }^{4}$ Benue State in north central Nigeria has the highest HIV burden with a sero -prevalence of $12.7 \%$ (Nigeria National Sentinel Survey, 2010). ${ }^{6}$ In 2001, the prevalence of HIV among TB patients in Nigeria, was estimated at $19.1 \%$ (Nigeria National Sentinel Survey, 2001). ${ }^{7}$ HIV is known to increase the burden of Tuberculosis and it is estimated that TB is the leading cause of death among People Living With HIV/AIDS (PLWHA). ${ }^{1,4}$ TB is believed to be responsible for 14-54\% of HIV/AIDS deaths globally (WHO Stop TB Dept. estimates 2002). ${ }^{1,4}$

TB management in the presence of HIV is complicated by several factors. The large pill burden could be confusing to the patient, association of the drugs to meals could also be a challenge while side effects may impede adherence. However, the most crucial challenge for the healthcare team responsible for the treatment of TB/HIV co-infection remains the drug-drug interactions between the rifamycins used to treat TB and the protease inhibitors (PI) and non-nucleoside reverse transcriptase inhibitors (NNRTIs) classes of drugs used to treat HIV infection. ${ }^{8}$ According to the Nigerian guidelines for the clinical management of TB and HIV/AIDS related conditions, Efavirenz is substituted for Nevirapine in the treatment of TB in co-infected patients because Rifampicin, a first line drug in the management of TB, lowers the serum concentration of Nevirapine. ${ }^{1}$ Previous recommendations specifically contraindicated the use of rifampicin with any of the Protease Inhibitors (PIs) or NNRTIs. However, new data indicate that rifampicin can be used for the treatment of active TB in patients whose antiretroviral regimen includes efavirenz with two or more NRTIs. A nevirapine- based regimen may be used in selected settings as well. ${ }^{8}$ Use of rifampicin with boosted saquinavir at any dose appears contraindicated. Rifabutin can be used with regimens containing efavirenz or nevirapine, or a single PI, except saquinavir alone, 
with some dose adjustments. Data is lacking on the use of rifabutin in antiretroviral regimens containing combinations of NNRTIs and PIs or multiple PIs and should be used with caution. In Nigeria, Rifabutin is neither readily available nor affordable further limiting the choice of rifamycins available for use.

The challenges of managing drug interactions and overlapping toxicities when treating co-infected patients makes it imperative that treatment of such patients are coordinated between the TB and HIV care providers to ensure optimal treatment for both diseases. It is important that health workers are on the alert for interactions between these two diseases hence the need to learn how to manage conditions arising from them competently.

This retrospective review aims to examine the level of compliance with substitution of efavirenz for Nevirapine in the management of TB/HIV co-infection.

\section{MATERIALS AND METHODS}

This was a retrospective study carried out at the highly populated HIV clinic of the Federal Medical Centre, Makurdi among HIV patients who were treated for tuberculosis. Makurdi is the capital of Benue, the state with the highest sero-prevalence of HIV in Nigeria located in north central Nigeria. All patients who registered for HIV care and treatment at the HIV clinic between January 2011 and December 2012 (2 years) were reviewed. The case notes of those patients who were treated for tuberculosis during the study period were retrieved and data on socio demographic characteristics and ARV drug regimen were extracted. Patients whose case notes had insufficient information were excluded. Data were entered into Microsoft Excel programme and imported into SPSS version 16 for analysis of proportions and percentages. The study was approved by the ethics committee of the hospital

III.

RESULTS

Four hundred and fifty patients were screened for TB. Out of these, 75 were found to have active TB giving a TB prevalence rate of $16.7 \%$. About one third (33.3\%) were aged 30-39 years, $22(29.3 \%)$ were 40 49years of age, $17(22.7 \%)$ were <30years and $11(14.7 \%)$ were $\geq 50$ years old. Majority $46(61.3 \%)$ of the patients were females. Over half $41(54.7 \%)$ had secondary or tertiary education and were single, divorced, separated or widowed. Only $28(37.3 \%)$ were on the national recommended Efavirenz based regimen for treating TBHIV co-infection, an equal number 27 (36\%) was on a Nevirapine based regimen while $2(2.7 \%)$ were on a protease inhibitor based regimen (Table 1)

Table 1: Socio-demographic characteristics and ARV regimen in treatment of TB/HIV co-infection

\begin{tabular}{lr}
\hline Sociodemography & $\mathbf{n}(\%)$ \\
\hline Age Group (Years) & $17(22.7)$ \\
$<30$ & $25(33.3)$ \\
$30-39$ & $22(29.3)$ \\
$40-49$ & $11(14.7)$ \\
$>50$ & \\
Gender & $46(61.3)$ \\
Female & $29(38.7)$ \\
Male & \\
Education & $24(32.0)$ \\
None & $10(13.3)$ \\
Primary & $30(40.0)$ \\
Secondary & $11(14.7)$ \\
Tertiary & \\
Marital status & $2(2.7)$ \\
Divorced & $29(38.7)$ \\
Married & $11(14.7)$ \\
Single & $12(16.0)$ \\
Separated & $16(21.3)$ \\
Widowed & \\
Drug Regimen & $28(37.3)$ \\
Efavirenz based & $27(36.0)$ \\
Nevirapine based & $2(2.7)$ \\
Protease inhibitor based & \\
\hline
\end{tabular}

\section{DISCUSSION}

The prevalence of TB among HIV patients in our study was $16.7 \%$. This is similar to overall national prevalence of $19.1 \%$ recorded in the Nigerian TB guidelines but it is lower than the prevalence of $32.8 \%$ reported by Awoyemi et $\mathrm{al}^{9}$ in a study of $58 \mathrm{HIV}$ positive adults at the university College Hospital, Ibadan, South-western Nigeria. The higher prevalence in the Ibadan study could have been because of a higher 
diagnostic yield from the addition of sputum culture as a diagnostic modality compared to our study which used only clinical features, chest radiographs and sputum AFB.

More females were seen in this study consistent with reports by several other workers who documented higher prevalence of HIV among women ${ }^{6,7}$ over half of those studied had secondary school education and above probably because the HIV treatment Centre is located in the state capital.

Only one third of those treated for TB were on the recommended Efavirenz based antiretroviral regimen for treatment of TB/HIV co-infection, another third were on a Nevirapine based regimen while $2.7 \%$ were on a PI based regimen.

The low compliance with the TB National guidelines recorded in our study could affect the outcome of treatment for both TB and HIV. Our finding is similar to the observation by Saraceni et al ${ }^{10}$ who noted non compliance with TB prophylaxis guidelines in brazil and opined that efforts to improve TB control in HIV patients were badly needed. Non compliance with guidelines could be a consequence of poor understanding of the guidelines by the attending clinicians or a communication gap between the wards, the DOTS clinic and the HIV clinic. Such lack of coordination could result in failure of substitution of Nevirapine for Efavirenz when patients are commenced on TB treatment considering that the attending physician at the HIV clinic may not be aware of the TB treatment commenced on the ward or at the DOTS centre. The consequences of non compliance can only be elucidated in further studies with larger subjects that would compare outcomes.

\section{CONCLUSION}

This study showed very low compliance with the Nigerian treatment protocol for TB/HIV co-infection. Efforts by all stakeholders to improve TB control in HIV patients are needed to significantly address the challenges of managing drug interactions and overlapping toxicities towards better treatment outcomes.

\section{REFERENCES}

[1]. Federal Ministry of Health. Department of Public Health. Guidelines for the clinical management of TB and HIV/AIDS in Nigeria. Abuja 2008.

[2]. WHO. AIDS, TB and Malaria. http://www.afro.who.int/en/nigeria/country-programmes/aids-tb-and-malaria.html. (Accessed May 30, 2013)

[3]. Sharma SK, Mohan A, Kadhiravan T.. HIV-TB co-infection: epidemiology. diagnosis and management. Indian J Med Res. 2005 Apr;121(4):550-67. [PubMed]

[4]. WHO. TB/HIV. http://www.who.int/tb/challenges/hiv/en/index.html. (Accessed May 30, 2013)

[5]. De Carvalho BM, Monteiro AJ, Neto RJP, Grangeiro TB, Frota CC. Factors related to HIV/ TB coinfection in a Brazilian Reference Hospital. BJID. 2008;12(4):281-286. [PubMed]

[6]. Federal Ministry of Health. Department of Public Health. National AIDS/STI Control Programme. Technical report on the 2010 National HIV Sero-prevalence Sentinel Survey. Abuja 2010.

[7]. Federal Ministry of Health. Department of Public Health. National AIDS/STI Control Programme. Technical report on the 2001 National HIV Sero-prevalence Sentinel Survey. Abuja 2001.

[8]. Munsiff S, Nilsen D, Ahuja S.D, Burzynski J.N. Antiretroviral Drugs and the Treatment of Tuberculosis. NYC Department of Health \& Mental Hygiene, Bureau of Tuberculosis Control. 2007;3-19

[9]. Awoyemi O B, Ige O M, Onadeko B O. Prevalence of active pulmonary tuberculosis in human immunodeficiency virus seropositive adult patients in University College Hospital, Ibadan, Nigeria. Afr J Med Med Sci. 2002 Dec ;31 (4):329-32

[10]. Saraceni V, Pacheco A.G. Physician adherence to guidelines for tuberculosis and HIV care in Rio de Janeiro, Brazil. Braz J Infect Dis $2011 ; 15(3): 249-252$ 